\title{
Residual elastic strain due to laser shock peening: synchrotron diffraction measurement
}

A M Korsunsky ${ }^{1 *}, \mathrm{~J} \mathrm{Liu}^{1}$, D Laundy ${ }^{2}$, M Golshan $^{2}$, and K Kim ${ }^{1}$

${ }^{1}$ Department of Engineering Science, University of Oxford, Oxford, UK

${ }^{2}$ Daresbury Laboratory, Synchrotron Radiation Department, Warrington, UK

The manuscript was received on 24 May 2005 and was accepted after revision for publication on 18 November 2005.

DOI: 10.1243/03093247JSA139

\begin{abstract}
Residual elastic strain in a thin slice parted off from a laser shock peened plate of titanium alloy Ti-6Al-4V was measured using high-energy diffraction on station 16.3 at SRS Daresbury. Diffraction peaks were collected for reflections (00.2), (10.1), (10.2), and (11.0) from the hcp (hexagonal close-packed) $\alpha$-phase of the titanium alloy. Reference values of the lattice spacing for each of the reflections were found from the diffraction pattern collected from a stress-free sampling volume. The residual elastic strain values calculated on the basis of each reflection were then computed and plotted as a function of distance from the treated surface. Residual strain profiles show significant differences, reflecting the variation in the elastic and plastic with orientation of the grain, i.e. anisotropy. The average macroscopic residual elastic strain was therefore computed using an appropriate average, taking into account multiplicity of each reflection. Since the greatest contrast in elastic and plastic properties exists between directions (00.2) and (11.0), the difference between residual elastic strains measured for these reflections was plotted, together with the 'difference strain' between (00.2) and (10.1). These showed a very good correlation with the plastic strain profile introduced by laser shock peening.
\end{abstract}

Keywords: energy-dispersive diffraction, synchrotron, strain mapping

\section{INTRODUCTION}

Laser shock peening is an advanced surface treatment technique aimed at introducing high levels of compressive residual stresses millimetres deep into the material surface, in order to enhance the resistance of engineering components to near-surface fatigue crack initiation and propagation. The method represents an evolution of other surface treatment techniques, such as shot and glass bead peening, low plasticity burnishing, induction hardening, etc. The common principle of all such techniques lies in the introduction of extensional plastic strains in the directions parallel to the treated surface by some inelastic deformation process.

A high-power laser beam is used to deliver energy densities in the order of several $\mathrm{GW} / \mathrm{cm}^{2}$ in a thin layer of ablative substance, e.g. polymer, water, etc.,

* Corresponding author: Department of Engineering Science, University of Oxford, Parks Road, Oxford OX1 3PJ, UK. email: alexander.korsunsky@eng.ox.ac.uk deposited on the sample surface. These laser beam energy densities are sufficient to create a small volume of rapidly expanding, high-pressure plasma between the coating and the substrate. This gives rise to a shock wave travelling into the substrate in the direction normal to the sample surface, causing plastic deformation in the near-surface layers. These plastically deformed layers (stretched in the plane parallel to the surface) remain attached to the underlying substrate. This implies that the residual elastic strain (and hence stress) in the nearsurface regions turns out to be compressive, and is balanced by the residual elastic tension in the deeper layers [1-3]. The total residual elastic strain in the final equilibrium condition everywhere in the sample must not exceed the elastic limit strain of the material.

If the component is subjected to an external loading cycle and remains elastic throughout, then the effect of residual compressive stress is to reduce the maximum tensile stress and thus to inhibit crack initiation [1]. If externally applied loading induces 
plasticity, then residual stress will be reduced or otherwise modified. For cyclic loading conditions it is the cyclic plastic strain that ultimately leads to crack initiation. It is obvious from the above discussion that the presence of residual stress modifies the cyclic plastic strain and thus affects crack initiation life.

Short fatigue cracks associated with localized plastic slip are known to be orientated so as to experience primarily shear loading. However, under continued cyclic loading cracks tend to turn in the direction perpendicular to the greatest applied tensile stress. In the case considered this usually corresponds to cracks growing perpendicular to the surface. As a consequence, any stress applied remotely is diminished by the presence of compressive residual stress, thus inhibiting crack propagation.

In order to predict the beneficial effect of laser shock peening on crack retardation, e.g. with the help of crack growth simulations using the Paris law, it is essential to have a precise knowledge of the residual stress profile and of the material property modification (e.g. hardening) induced by the treatment. In the present study the discussion is focused on the method and results of residual strain measurement carried out using the non-destructive technique of high-energy synchrotron X-ray diffraction $[2,3]$.

Synchrotron radiation provides a high-resolution probe for obtaining information on stress and strains deep in the bulk of engineering components and structures. It has become a mature instrument for the determination of residual stress states in small coupons, and developments are under way to establish the facilities for performing high-resolution measurements directly on larger engineering components. This approach holds out the prospect of furnishing a validation tool for tuning-up manufacturing processes so as to reduce distortion and residual stress, and to increase the durability of components [3].

The purpose of the present study was twofold: (a) to collect residual elastic strain profiles across a thin plate parted off from a laser shock peened plate of Ti64 alloy and (b) to extract information about the elastoplastic deformation of polycrystalline hcp (hexagonal close-packed) $\alpha$-phase of the titanium alloy from these data.

Titanium alloys are widely used in the aviation industry due to their excellent combination of low density combined with their stiffness, strength, and ductility. Hence, understanding the behaviour of this material when subjected to a variety of manufacturing processes, i.e. rolling, quenching, shot peening, and laser shock peening, is crucial in structural integrity of the components where titanium alloys are used.

The paper is organized as follows. Firstly, the fundamentals of deformation behaviour of polycrystalline aggregates are reviewed. Next, the basic methods of lattice parameter determination by diffraction are briefly summarized. Then the experimental set-up and data collection procedures are presented that were used on Station 16.3 at SRS Daresbury, and the procedures used for interpreting the results are discussed. Finally, the results are reviewed in terms of their significance for understanding the mechanical response of the polycrystalline material used in the present study and judgement is made about the permanent plastic strain introduced by the process. The analysis of the complete stress state generated by the laser shock peening procedure is presented in a companion paper [4].

\section{FUNDAMENTALS OF POLYCRYSTALLINE ELASTOPLASTIC DEFORMATION}

Many metallic alloys are used for load-bearing applications in polycrystalline form. The deformation of polycrystalline aggregates has therefore attracted wide interest and resulted in a large number of theoretical and experimental studies. At least two distinct modes of description traditionally used can be identified: macroscopic continuum modelling, which usually assumes material to be isotropic and homogeneous, and anisotropic, non-uniform elastoplastic description, capable of taking into account the difference in deformation between individual crystallites. The former description provides a very efficient tool for dealing with macroscopic objects at the 'engineering' scale, but fails to account for the stiffness and strength mismatch between different grains at the microscopic scale, although it is apparent that in fact these are likely to be crucially responsible for many modes of material failure. The difficulty in using the microscopic crystal description in its fullness is that the number of individual grains that have to be modelled is extremely large, perhaps on the order of $10^{10}$ within a medium-sized specimen. Even at the present level of computer hardware development, modelling the deformation of every grain in a small sample remains a great challenge.

To overcome this difficulty attempts have been made to concentrate on the deformation of a representative volume element (RVE) and then to generalize the result on to the entire macroscopic object. One of the principal challenges that immediately arise in this context is the necessity to maintain 
material continuity and stress balance conditions between grains of different phases and orientations. Different approaches have been proposed. If one aspect of the continuity requirements is ignored, then upper or lower bound approximations to the correct solution arise. For example, assigning constant uniform strain in all grains enforces strain compatibility in the crudest sense. This approximation is associated with the name of Voigt, and provides the upper bound (stiff) description of the response. The grain-to-grain variation of the stress state can be considered, but stress continuity between neighbouring grains is violated. Alternatively, it is possible to assume that all grains experience the same stress, but different strain depending on their stiffness. This approximation is associated with the name of Reuss, and provides a lower bound (compliant) description of the response. Sachs published one of the early studies on the subject [5] in which the above approaches were discussed. Sachs used the uniform strain Voigt approximation to consider not only elastic but also plastic behaviour of a polycrystalline aggregate on the basis of the Schmid law of crystal slip [6].

A more accurate solution to the problem with elastoplastic deformation of a polycrystalline aggregate lies between the simple upper and lower bounds of Voigt and Reuss. Several strategies have been proposed. One such strategy relies on the formulation of self-consistent elastoplastic deformation models whereby each grain must deform so as to preserve both stress continuity and strain compatibility with the surrounding material [7]. The grain is considered to be ellipsoidal, and the surrounding material is thought to be a uniform continuum with the properties obtained as the average of the response of all other grains [8]. This family of models is highly efficient and well-suited for resolving the effects at the intermediate scale between continuum and local microscopic deformation behaviour, e.g. at the level of averages across grain groups sharing common orientations. This level of description is highly appropriate to diffraction data interpretation $[9,10]$.

The self-consistent model described above is not aimed at capturing the details of local deformation, say, between two neighbouring grains of particular orientations. For this purpose finite element polycrystal plasticity models have been developed [11] that allow local effects to be captured. Although these models require a much greater computational effort than self-consistent models, the large amounts of detailed additional information that they provide serves as justification.
In the material considered in the present study two principal phases are present, the majority hcp $\alpha$-phase, and the minority bcc (body-centred cubic) $\beta$-phase. The $\beta$-phase is capable of plastic deformation by restricted slip (pencil glide) along the closely packed [111] direction, while the $\alpha$-phase is deformed readily both by twinning and slip on basal, prismatic, and pyramidal planes, depending on the nature of deformation (tension or compression), strain rate, and temperature. If two neighbouring grains are considered belonging to different phases, and hence having different stiffness and plastic strength, then under remotely applied load the stronger and stiffer phase is likely to carry a higher proportion of the load while experiencing lower strain.

The same argument is valid for neighbouring grains of the same phase that have different orientation with respect to the applied load [12]. In the present study attention is focused on the deformation of the majority $\alpha$-phase. In the hcp lattice cell of this material the extremes of elastic and plastic properties, i.e. stiffness and yield strength respectively, are exhibited by the (002) and (110) planes illustrated in Fig. 1. The easiest mode of plastic deformation in $\alpha$-titanium is basal slip within the (002) type planes. Grains loaded in the direction normal to the (002) planes exhibit higher yield strength, since the critical resolved shear stress on these planes vanishes. In contrast, easy slip is possible if the grain is loaded in the direction perpendicular to the (110) plane. The reflections corresponding to these two types of lattice plane are expected to show extremes of behaviour.

The purpose of the present study was to use the insight into the elastoplastic polycrystalline deformation to help inform the experimental techniques so as to allow the interrogation of the behaviour of grain groups sharing similar orientation by diffraction. Key requirements for these techniques

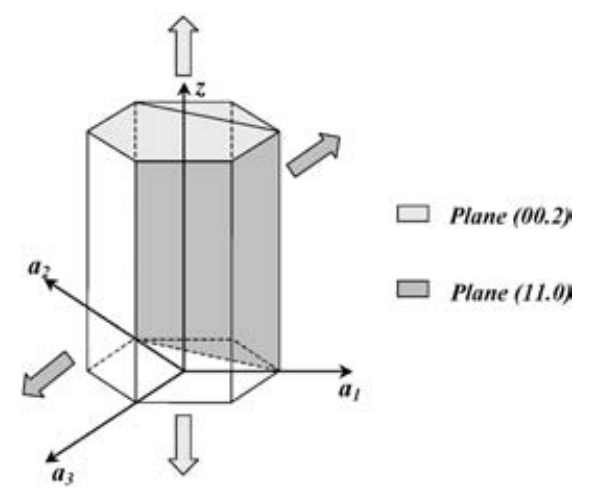

Fig. 1 Some orientations of lattice planes within the hcp unit cell 
are (a) to produce an estimate of the average macroscopic elastic strain that is used to compute the 'engineering' stress, (b) to identify the amount of plastic deformation experienced by the material, and (c) to highlight differences in the strains experienced by grains of different orientations.

\section{EXPERIMENTAL SET-UP AND DATA INTERPRETATION}

There are two principal methods for extracting residual elastic strain information within objects with the help of X-ray diffraction, namely the angle dispersive (monochromatic beam) technique and the energy-dispersive (white beam) technique.

In the monochromatic mode the beam is first passed through an optical element that transmits only photons with the energy or wavelength lying within a narrow bandwidth. The optical element in question (the monochromator) is usually a highly perfect crystal placed in the beam in reflection orientation (Bragg mode) or transmission orientation (Laue mode). The bandpass depends on the quality of the crystal and can be as narrow as $\Delta \lambda / \lambda=10^{-4}$. In many practical situations this accuracy may exceed requirements, and a broader bandpass is in fact beneficial, since it increases flux on the sample, the number of crystals within a polycrystalline sample that contribute to the pattern, etc.

In the present study a single-bounce bent Laue mode monochromator was used [13], as shown in Fig. 2. Bending the crystal serving as a Laue monochromator serves two purposes: on the one hand, bending induces tensile and compressive strains on different sides of the crystal, thus increasing the bandpass and hence the flux incident on the sample. In many engineering applications this is in fact an advantage, since peak broadening is dominated by sample effects (strain spread within the gauge volume), and no advantages are obtained by using extremely fine monochromation.

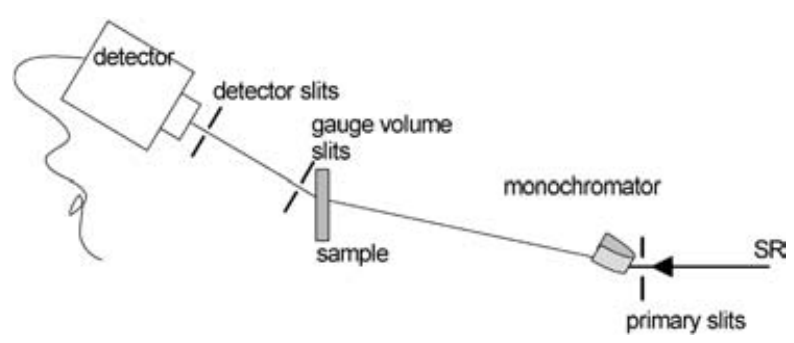

Fig. 2 Schematic diagram of a high-energy synchrotron X-ray diffraction experiment set-up using a bent Laue monochromator
Diffraction patterns are collected by employing a detector scanning the scattering angle $2 \theta$ or a position-sensitive detector capable of registering total photon flux simultaneously at several positions along a line or over a two-dimensional surface. This mode allows accurate determination of diffraction peak intensity, shape, and position. However, it usually requires significantly longer counting times in comparison with the white beam mode in order to collect the data from comparable sections of the diffraction pattern, primarily due to the reduction of flux by monochromation, but also due to the necessity of scanning the detector.

An energy dispersive set-up allows multiple diffraction peaks to be collected simultaneously, thus achieving particularly efficient counting statistics at energies above $30 \mathrm{keV}$ [14]. The accuracy of determination of individual peak position and shape resolution in the white beam mode is usually related to the resolution of the energy-dispersive detector, but can in fact be several orders of magnitude better [14]. The accuracy of interpretation in terms of lattice parameters and hence strain can be significantly improved by using multiple peak analysis or whole pattern fitting [13].

The material used in the present study was Ti-6Al-4V alloy, which is widely used to manufacture components located at the front of the aeroengine, such as fan and compressor blades and discs; it also has many other structural applications. Production comprises vacuum arc melting above the beta transus phase followed by plate rolling in order to minimize the crystallographic texture in the material, which results in anisotropy of both elastic and inelastic properties. The structure achieved by the manufacturing process is obtained by solutiontreating in the middle of the $\alpha+\beta$-phase field and air cooling. This gives a mixture of primary alpha and a transformation product that comprises the $\alpha$ - and $\beta$-phases. The $\alpha$-phase is an hcp structure, which is typical of titanium at room temperature and transforms to a bcc structure, $\beta$-phase, at $883^{\circ} \mathrm{C}$. The size of the grains is approximately $5 \mu \mathrm{m}$.

Laser shock treatment was performed by an Nd:YAG (neodymium-doped yttrium aluminium garnet) laser (wavelength $355 \mathrm{~nm}$ ) at the energy density of approximately $7 \mathrm{GW} / \mathrm{cm}^{2}$, spot size of about $2 \times 3 \mathrm{~mm}^{2}$ and coverage of 200 per cent, with an impulse duration of about $20 \mathrm{~ns}$. A square $(50 \mathrm{~mm} \times 50 \mathrm{~mm})$ coupon was electrical discharge machined from a plate of Ti-64 alloy $h_{x}=8.5 \mathrm{~mm}$ thick that had been subjected to laser shock peen treatment on one of the surfaces.

The geometry of the specimens is illustrated in the schematic diagram in Fig. 3, together with the system 


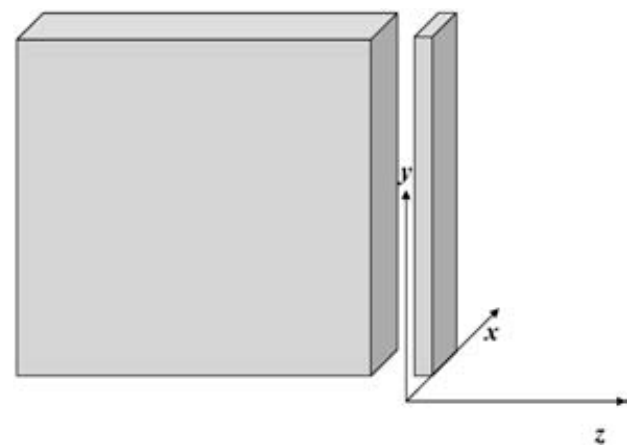

Fig. 3 Schematic illustration of the square coupon from the plate of Ti-64 alloy, and the slice parted off for the transmission diffraction investigation

of coordinates used for reference. In the diagram, the laser shock peened surface of the plate is the bottom surface corresponding to the plane $x=0$. An $h_{z}=4 \mathrm{~mm}$ thick slice was then parted off from the coupon using electrical discharge machining (EDM) to minimize any disturbance to the plastic strain distribution.

In the synchrotron strain measurement experiment the sample was mounted so that the incident beam direction corresponded to the negative $z$ axis. The Laue monochromator was used to select the energy of approximately $60 \mathrm{keV}$ from the white beam produced by the wavelength shifter. The wavelength of the monochromatic beam is determined from the photon wavelength using the simple formula

$$
\lambda(\AA)=\frac{12.39842}{E(\mathrm{keV})}
$$

The detector was set to perform an angular scan in the vertical diffraction plane around the $x$ axis. In the course of the scan the orientation of the slice within the diffraction plane was also adjusted, in such a way that the angle $\theta$ of rotation of the slice around the horizontal $x$ axis corresponded to half of the diffraction angle, $2 \theta$. Coupled $\theta-2 \theta$ scan ensured that the orientation of the scattering vector always coincided with the sample $y$ axis along the longest slice edge in the diagram of Fig. 3.

The incident beam spot was set using collimating slits to the size of $s_{x}=25 \mu \mathrm{m}$ by $s_{y}=100 \mu \mathrm{m}$. This selection was made on the basis of the following requirements. Firstly, a high spatial resolution with the distance from the laser shock peened surface is required in order to obtain adequate description of the near-surface residual elastic strain field; hence $s_{x}=25 \mu \mathrm{m}$. The beam dimension $s_{y}=100 \mu \mathrm{m}$ in the vertical diffraction plane, on the other hand, can be made larger, since all the points contained within the gauge volume extended in the direction parallel to the laser shock peened surface experience similar residual elastic strain. At depths beyond about $2.5 \mathrm{~mm}$, where the strain gradients are expected to be lower and the requirements of spatial resolution are therefore less stringent, both the width of the beam spot $s_{x}$ and the scanning step were increased to $100 \mu \mathrm{m}$.

For each position of the gauge volume within the sample, detector scans were performed over several angular ranges so as to collect reflections (00.2), (10.1), (10.2), and (11.0) from the hcp $\alpha$-phase of the titanium alloy. Unstrained peak data for each of these reflections was collected by performing a similar measurement for the gauge volume located at the very corner (say, near $x=0, y=0$ ) of the sample. This choice of reference was based on the argument that the sampling volume in such a position must be free from tractions (and hence stresses) in the $x$ and $y$ directions. The only stress component that may be present in such a volume is therefore the $z$ component, but that component is also strongly relieved by the traction-free requirement at upstream and downstream slice surfaces $z=0 \mathrm{~mm}$ and $z=4 \mathrm{~mm}$.

The gauge volume should therefore be free from macroscopic average stress, and hence free from macroscopic average residual elastic strain. It should be noted that the above argument does not quite prevent the 'corner' gauge volume from containing microscopic residual stresses that exist between grains of different orientations.

Peaks were fitted using Gaussian shape functions to determine their intensity, width, and peak centre position. Residual elastic strain values were calculated using the following standard formulae

$$
d=\frac{\lambda}{2 \sin \theta}, \quad \varepsilon=\frac{d-d_{0}}{d_{0}}
$$

Strain values at each scanning position of the sample were calculated based on individual reflections. This provided the data for compiling reflection-specific strain profiles across the sample, which are presented and discussed in the next section.

\section{RESULTS AND ANALYSIS}

Figure 4(a) illustrates the strain profiles computed using reflections (00.2) and (11.0). Two observations can immediately be made from the plots shown. Clearly, both residual elastic strain profiles tell the same story. Laser shock peened material close to the surface finds itself in the state of residual elastic compression, following the plastic stretching experienced 


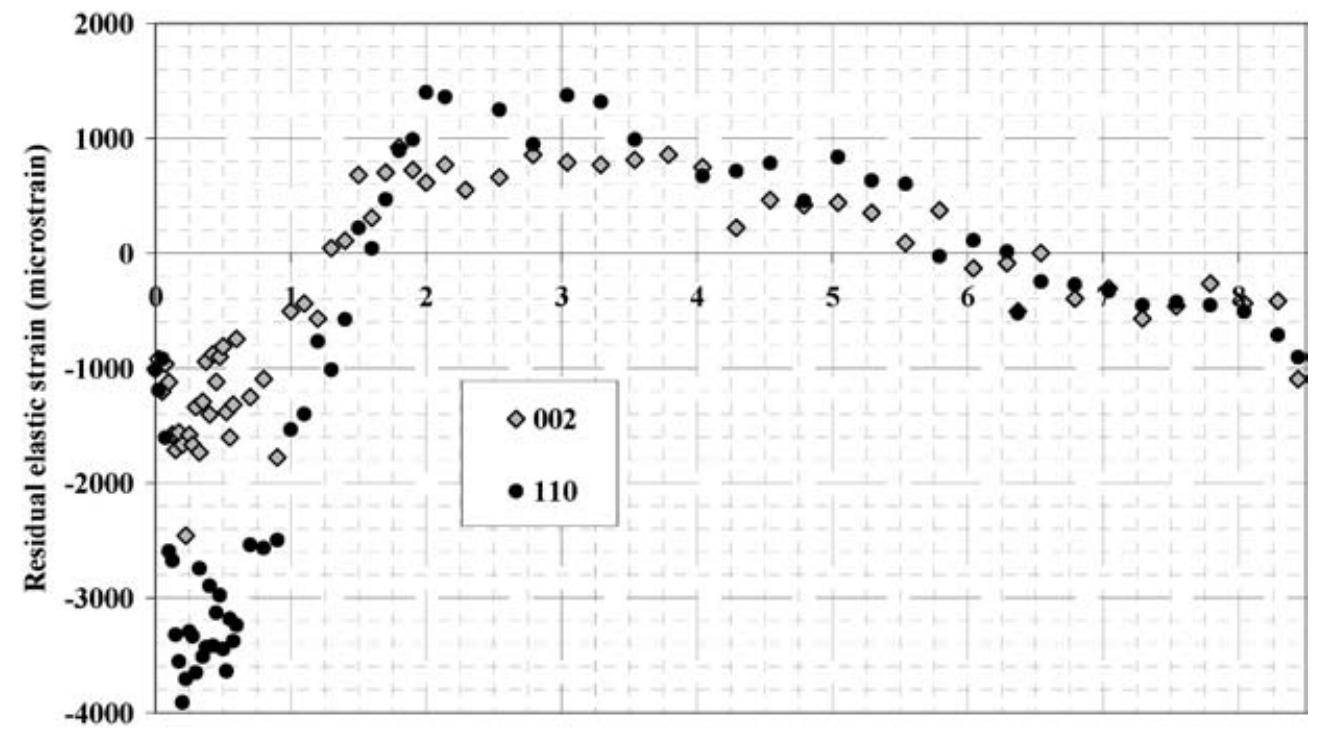

(a)

Position $\times(\mathrm{mm})$

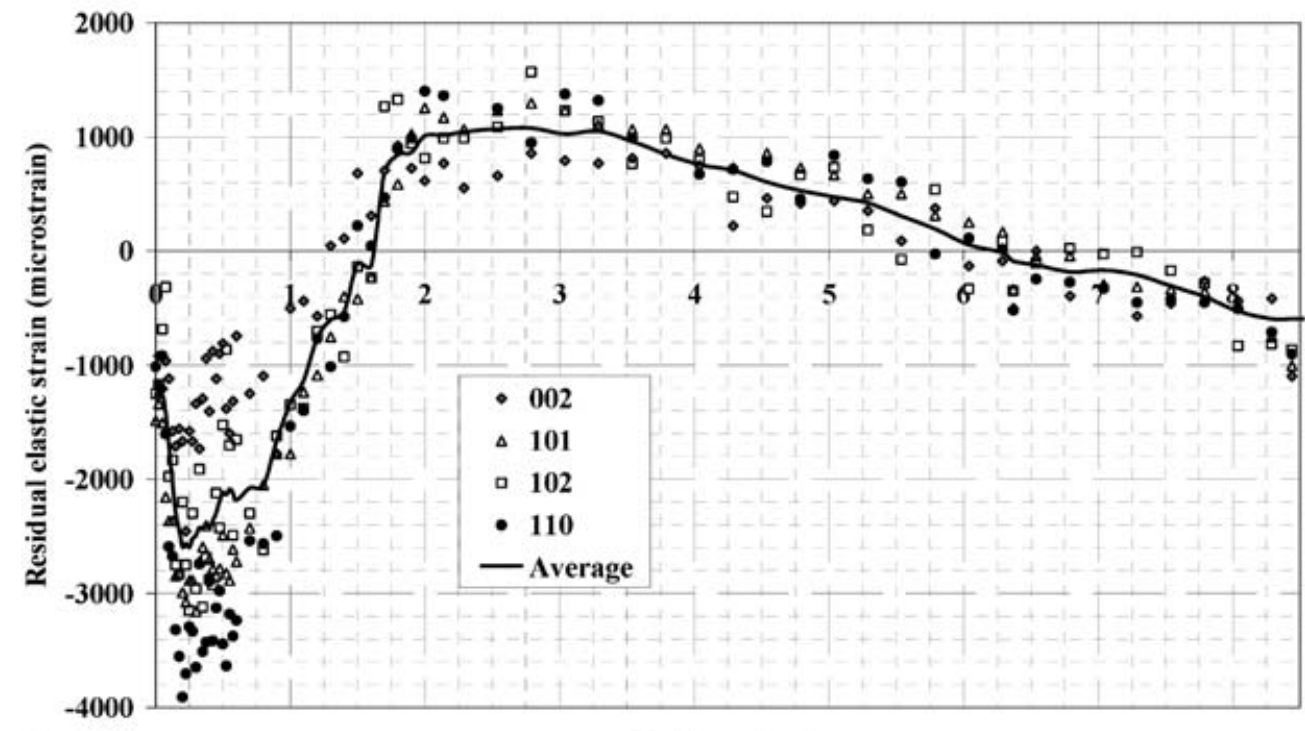

(b)

Position x (mm)

Fig. 4 (a) Profile of residual elastic strains for reflections (00.2) and (11.0) parallel to the laser shock peened surface plotted as a function of distance from the treated surface across the entire sample depth. (b) Profile of the residual elastic strain parallel to the laser shock peened surface plotted as a function of distance from the treated surface across the entire sample depth. Markers indicate the strain values computed using individual diffraction peaks (00.2), (10.1), (10.2), and (11.0). The line indicates an estimate of the macroscopic average residual elastic strain computed using appropriate weighting of the available peak strains

during peening. The depth of material directly affected by laser shock peening treatment is close to $2 \mathrm{~mm}$.

The two strain profiles considered show very significant discrepancies, both close to the laser shock peened surface and deeper in the sample, confirming that the polycrystalline material studied possesses a large degree of anisotropy, both in the elastic and plastic sense. To illustrate this point further, Fig. 4(b) shows the collection of four individual elastic lattice strain profiles, for reflections (00.2), (10.1), (10.2), and (11.0). It is apparent that the trends of variation are similar for all the strains, but that individual responses vary very significantly. This observation has strong implications for experimental stress evaluation by diffraction using the $\sin ^{2} \Psi$ method. For obtaining the stress values, elastic stiffness constants are used, in conjunction with the orientational dependence of 
the diffraction strain corresponding to a particular reflection. Figure 4(b) provides an illustration of why these constants sometimes display such strong dependence on the reflection, and how important it is to identify and use their correct value in each particular case. It is also possible to surmise that a single choice of X-ray elastic constants may not be equally suitable for the elastic and plastic regimes of deformation.

In order to obtain a confident estimate of the macroscopic average residual elastic strain within the gauge volume, it is best to use the strain data from as many reflections as possible. The logic behind this statement is simple: the sum of all reflections (representing orientational average of strains) with appropriate weights must converge to the macroscopic residual elastic strain value (by definition of the latter). Since in the present case only a limited number of reflections are available, these can be added up with weights determined by the multiplicity of the appropriate reflections, to obtain an approximation to the average value [15]. The result is plotted in Fig. 4(b) as the continuous line. This parameter provides the best data series for the purposes of engineering residual stress analysis.

At depths beyond $x=2 \mathrm{~mm}$ the residual strain profile appears to be close to linear, representing the elastic bending response to the incompatibility of strain induced by plastic deformation [16]. Detailed analysis of elastic deformation in the presence of inelastic strains has been presented in another publication [16]. Here it suffices to note that it was rigorously demonstrated that the total observed residual elastic strain is the sum of the eigenstrain (e.g. plastic strain) introduced into the piece by some inelastic deformation process, e.g. peening, plastic bending, creep, cutting and bonding, welding, etc., and the elastic deformation strain field arising in response to this process. Since in the present case the elastic response amounts simply to the elastic bending profile, it is easy to 'back out' the plastic strain profile by calculating the difference between the elastic bending line calculated by matching the deformation beyond $2 \mathrm{~mm}$ of depth and the measured profile.

The plastic strain calculated in this way is plotted in Fig. 5, multiplied by the factor of 0.5 for convenience of comparison (see below). Detailed analysis therefore suggests that plastic strain persists to depths up to $3 \mathrm{~mm}$, although it has significant values mainly at depths not exceeding $2 \mathrm{~mm}$ and reaches its maximum of 4500 microstrains at the depth of approximately $0.25 \mathrm{~mm}$ from the surface.

The other two data series with which the plastic strain is compared in Fig. 5 represent the difference strains $\left(\xi_{00.2}-\xi_{10.1}\right)$ and $\left(\xi_{00.2}-\xi_{11.0}\right)$. Several interesting observations can be made on the comparison. Firstly, it is clear that both difference strains show strong

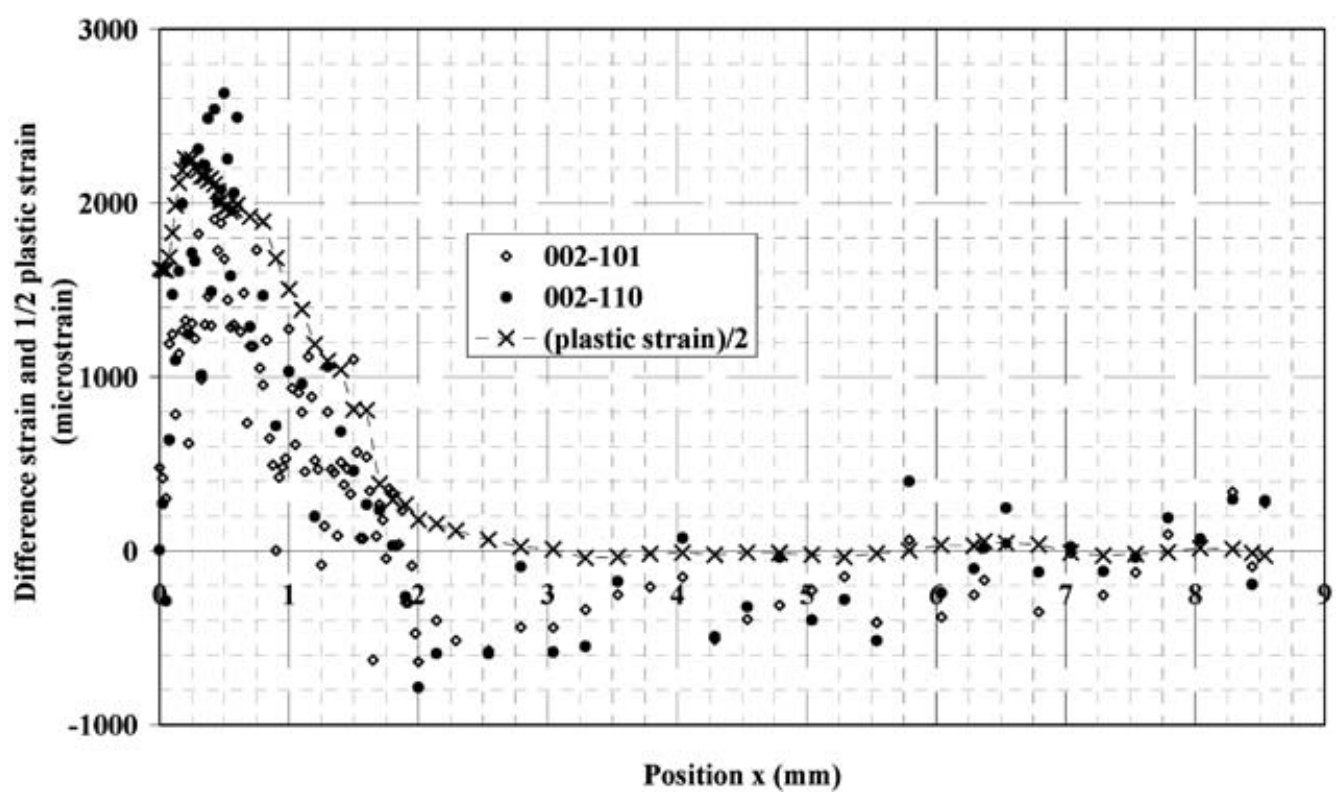

Fig. 5 'Difference' strain plotted as a function of depth from the laser shock surface. High values of the 'difference strain' indicate the region where significant plastic deformation took place. Also plotted (cross markers) is the plastic strain, worked out as the difference between the measured residual elastic strain profile and the 'pure bending' line, multiplied by a factor of 0.5 for ease of comparison 
correlation with the plastic strain distribution, displaying high values (up to 2600 microstrains) in the nearsurface region. However, whereas plastic strain falls to zero at depths exceeding about $3 \mathrm{~mm}$, difference strains continue to vary, following a somewhat linear trend. This indicates that difference strains respond both to plastic and elastic deformation, as expected from the analysis of elastoplastic deformation of polycrystalline aggregates presented in this paper. However, it is also clear that the response of difference strains to plastic deformation is much more dramatic than to elastic straining. Finally, it is worth noting that the difference between (00.2) and (11.0) peak strains displays the greatest sensitivity to plastic deformation, although the other difference strain pair, (00.2) and (10.1), also reveals a significant effect. These observations suggest that difference strains can serve as a useful indicator of plastic deformation history.

\section{CONCLUSIONS}

Data analysis and interpretation were presented for a residual elastic strains laser shock peened plate of Ti-64 titanium alloy. Measurements were performed using high-energy diffraction on station 16.3 at SRS Daresbury. Diffraction peaks collected from reflections (00.2), (10.1), (10.2), and (11.0) of the hcp $\alpha$-phase were used in order to estimate the macroscopic average residual elastic strain, to obtain an approximation to the plastic strain profile produced by laser shock peening, and also to identify 'difference strains' that provide information about plastic deformation history within the gauge volume.

\section{REFERENCES}

1 Ruschau, J. J., John, R., Thompson, S. R., and Nicholas, T. Int. J. Fatigue, 1999, 21, S199.

2 Martinez, S. A., Sathish, S., Blodgett, M. P., and Shepard, M. J. Expl Mechanics, 2003, 43, 141.

3 Chen, H. Q., Yao, Y. L., and Kysar, J. W. Trans. ASME, J. Mfg Sci. Engng, 2004, 126, 226.

4 Korsunsky, A. M., Regino, G. M., and Liu, J. J. Strain Analysis, 2005 (submitted).

5 Sachs, Z. Ver. Dtsch. Ing., 1928, 72, 734.

6 Schmid, E. and Boas, W. Kristallplastizität, 1935 (Springer-Verlag, Berlin).

7 Hutchinson, J. W. Proc. R. Soc. Lond., 1970, A319, 247.

8 Turner, P. A. and Tome, C. N. Acta Mater., 1994, 42, 4143.

9 Clausen, B., Lorentzen, T., Bourke, M. A. M., and Daymond, M. R. Mater. Sci Engng A - Struct. Mater. Properties, Microstruct. and Processing, 1999, 259, 17.

10 Korsunsky, A. M., James, K. E., and Daymond, M. R. Intergranular stresses in polycrystalline fatigue: diffraction measurement and self-consistent modelling. Engng Fract. Mechanics, 2004, 71, 805-812.

11 Busso, E. P., Cailletaud, G., and Dunne, F. Int. J. Plasticity, 2001, 17, 409.

12 Korsunsky, A. M., Daymond, M. R., and James, K. E. Mater. Sci. Engng A - Struct. Mater. Properties, Microstruct. and Processing, 2002, 334, 41.

13 Liu, J., Kim, K., Golshan, M., Laundy, D., and Korsunsky, A. M. J. Appl. Cryst., 2005, 38, 661-667.

14 Korsunsky, A. M., Collins, S. P., Owen, R. A., Daymond, M. R., Achtioui, S., and James, K. J. Synch. Rad., 2002, 9, 77-81.

15 Daymond, M. R. J. Appl. Phys., 2004, 96, 4263-4272.

16 Korsunsky, A. M. J. Strain Analysis, 2005, 40, 817-824. 